\title{
Attributes Weight Determination in Magdm Problems using Some Numerical Method Techniques
}

\author{
Akila .S, John Robinson .P
}

\begin{abstract}
Here MAGDM problems are explored. Attribute weight data given are in the form of problems involving numerical method solutions. The decision information during this study is in the shape of Intuitionistic Fuzzy Numbers (IFNs) derived from Intuitionistic Fuzzy Sets (IFSs). The IFOWA operator and IFHA operator are used for collecting the combined matrix. The modified Euler-Cauchy method and Heun method are used to calculate the unidentified weights. Effectiveness of the projected method is established using Numerical Illustrations.

Keywords- Intuitionistic Fuzzy Sets(IFS), Intuitionistic Fuzzy Value(IFV), IFWA operator, IFOWA operator, IFHA operator, MAGDM, Numerical Methods, Euler and Heun Method.
\end{abstract}

\section{INTRODUCTION}

MAGDM problems are mostly used in Engineering, Management and Economics field. It also act as an essential part in actual life. Problem for sorting the most reasonable way to find in an finite number of possible alternatives evaluated on multiple attributes. Generally the human decision making includes the information which should mentioned in details of intuitionistic fuzzy. Now a days the researchers make research on Intuitionistic Fuzzy environment of MAGDM Problems. Atanassov (1986. 1994 1999) projected Intuitionistic Fuzzy set. Problems in MAGDM are used in IFS and Triangular Intuitionistic Fuzzy Sets as an applications of Sumudu Transform by Jeeva \& Robinson, (2017, 2018). Robinson \& Indhumathi, (2018) developed in Intuitionistic Fuzzy MAGDM Problems on differential equations of Singularly perturbed. Kingston, Robinson \& Dhayabaran, (2016) solved the Group Decision Making Problems of Multiple Attribute with Runge-Kutta Method of Harmonic Mean. Gerstenkorn \& Manko, (1991) introduced a distinct Correlation coefficient method to derive the interrelation of IFS. The values of correlation coefficient lies between 0 and 1. Robinson \& Amirtharaj, $(2012,2014)$ Vague sets and Interval Vague sets correlation coefficient are proposed.

To sort Numerical approximations to the solutions of Ordinary Differential Equations numerical methods are used. When the first derivative of the variables alone appears in the equation then it is a first order differential equation in the Initial Value Problem. Jain et al. (1999) has solved interpolation and approximation, Differentiation and Integration problems using various methods. Mathews (2012) used MATLAB Software for solving the Numerical methods which simplifies the human effort in calculating. For aggregating the information different type of aggregation operators have been found. Most frequent aggregation operators are Ordered Weighted Average Operator which was introduced by Yager, (1988). He also introduced Generalized, Fuzzy Ordered Weighted Average operator. Yager, \& Filev, (1999) have proposed Induced ordered weighted averaging operators. Yager, $(1988,2004)$ also has developed Generalized OWA aggregation operators. Xu, (2005) defines Intuitionistic Preference relations and investigated the intuitionistic fuzzy Multiple Attribute Decision Making with the information about attribute weights completely unknown. In this paper, some numerical methods are adopted for calculating the unknown decision maker weights in the decision problem, the intuitionistic fuzzy numbers are based on the decision data. The efficiency of planned method is specified through Numerical illustration.

\section{Definition I:}

Here $\tilde{a}_{j}=\left(\mu_{j}, \gamma_{j}\right), j=1,2, \ldots, n$ is the group of Intuitionistic Fuzzy Values. IFWA: $Q^{n} \rightarrow Q$ expressed by

$\operatorname{IFWA}\left(\tilde{a}_{1}, \tilde{a}_{2}, \ldots, \tilde{a}_{n}\right)=\sum_{j=1}^{n} \omega_{j} \tilde{a}_{j}=\left(1-\prod_{j=1}^{n}\left(1-\mu_{j}\right)^{\omega_{j}}, \prod_{j=1}^{n} \gamma_{j}^{\omega_{j}}\right)$

Where $\omega=\left(\omega_{1}, \omega_{2}, \ldots, \omega_{n}\right)^{T}$ be the weight vector of $\tilde{a}_{j}$,

in each $j=1,2, \ldots, n, \omega_{j}>0$ and $\sum_{j=1}^{n} \omega_{j}=1$.

Definition II:

Here $\tilde{a}_{j}=\left(\mu_{j}, \gamma_{j}\right)$, for all $j=1,2, \ldots, n$ in group of IFV

IFOWA: $Q^{n} \rightarrow Q \quad$ explained as:

$\operatorname{IFOWA}_{w}\left(\tilde{a}_{1}, \tilde{a}_{2}, \ldots, \tilde{a}_{n}\right)=\sum_{j=1}^{n} w_{j} \tilde{a}_{\sigma(j)}=\left(1-\prod_{j=1}^{n}\left(1-\mu_{\sigma(j)}\right)^{w_{j}}, \prod_{j=1}^{n} \gamma_{\sigma(j)}^{w_{j}}\right)$.

Published By:

Blue Eyes Intelligence Engineering

\& Sciences Publication 


\section{ATTRIBUTES WEIGHT DETERMINATION IN MAGDM PROBLEMS USING SOME NUMERICAL METHOD TECHNIQUES}

$w=\left(w_{1}, w_{2}, \ldots, w_{n}\right)^{T}$ is associated weighting vector for that $w_{j}>0, \quad \sum_{j=1}^{n} w_{j}=1 . \quad$ Furthermore, $(\sigma(1), \sigma(2), \ldots, \sigma(n))$ is a permutation of $(1,2, \ldots, n)$, in it $\tilde{a}_{\sigma(j-1)} \geq \tilde{a}_{\sigma(j)}$ each $j=2, \ldots, n$.

Definition III:

Let $a_{j}=\left(\mu_{j}, v_{j}\right)$, every $\mathrm{j}=1,2,3, \ldots, \mathrm{n}$ is a group of IFV

IFHA : $\mathrm{Q}^{\mathrm{n}} \rightarrow \mathrm{Q}$,

$I F H A_{\omega, w}\left(a_{1}, a_{2}, \ldots, a_{n}\right)=\sum_{j=1}^{n} a_{\sigma(j)} w_{j}=\left[1-\prod_{j=1}^{n}\left(1-\mu_{a_{\sigma(j)}}\right)^{w_{j}}, \prod_{j=1}^{n}\left(v_{a_{\sigma(j)}}\right)^{w_{j}}\right]$

$\mathrm{w}=\left(\mathrm{w}_{1}, \mathrm{w}_{2}, \ldots \ldots, \mathrm{w}_{\mathrm{n}}\right)^{\mathrm{T}}$ is linked vector in which $\mathrm{w}_{\mathrm{j}}>0$ and $\sum_{j=1}^{n} w_{j}=1$,

$\omega=\left(\omega_{1}, \omega_{2}, \ldots \ldots, \omega_{n}\right)^{\mathrm{T}}$ to be weight vector of $\mathrm{a}_{\mathrm{j}}$, for all $\mathrm{j}=1,2, . ., \mathrm{n}$ such that $\omega_{\mathrm{j}}>0$ and $\sum_{j=1}^{n} \omega_{j}=1 . a_{\sigma(j)}$ are $\mathrm{j}^{\text {th }}$ biggest in weighted. IFNs $a_{j}=a_{j}^{n \omega_{j}}, \mathrm{j}=1,2, . ., \mathrm{n}$.

\section{TO FORMULATE GROUP DECISION MAKING ALONG INTUITIONISTIC FUZZY INFORMATION \& RESULTS}

Step 1:

Using the information specified in the decision matrix of Intuitionistic fuzzy $\tilde{R}_{k}$ and the IFOWA Operator, $\tilde{r}_{i}^{(k)}=\left(u_{i}^{(k)}, v_{i}^{(k)}\right)=\operatorname{IFOWA}=\left(\tilde{r}_{i_{1}}^{(k)}, \tilde{r}_{i_{2}}^{(k)}, \ldots, \tilde{r}_{i_{n}}^{(k)}\right), i=1,2$

For solving the individual overall preference in IFV $\tilde{r}_{i}{ }^{k}$ of the substitute $A_{i}$.

Step 2:

Using IFHA operator,

$\tilde{r}_{i}=\left(\mu_{i}, \gamma_{i}\right)=I F H A_{v, w}=\left(\tilde{r}_{i}^{(1)}, \tilde{r}_{i}^{(2)}, \ldots, \tilde{r}_{i}^{(t)}\right), i=1,2, \ldots m$

To obtain combined general preference IFV $\tilde{r}_{i}(i=1,2, \ldots . m)$ for alternative $\mathrm{A}_{\mathrm{i}}, \quad$ that $v=\left(v_{1}, v_{2} \ldots v_{n}\right)$ is weighting vector of decision makers, along $V_{k} \in[0,1], \quad \sum_{k=1}^{t} V_{k}=1 ; \quad w=\left(w_{1}, w_{2} \ldots w_{n}\right)$ are related weighting vector of IFHA Operator along $w_{j} \in[0,1], \quad \sum_{j=-1}^{n} w_{j}=1$

Step 3:

To find coefficient of correlation, here $\tilde{r}_{i}=(0,1)$, $\mathcal{A}, \mathcal{B} \in$ IFSs are given in rule

$$
\begin{gathered}
C_{z l}(\mathcal{A}, \mathcal{B})=\frac{1}{n} \sum_{i=1}^{n}\left[\mu_{\mathcal{A}}\left(x_{i}\right) \mu_{\mathcal{B}}\left(x_{i}\right)+\gamma_{\mathcal{A}}\left(x_{i}\right) \gamma_{\mathcal{B}}\left(x_{i}\right)+\right. \\
\left.\pi_{\mathcal{A}}\left(x_{i}\right) \pi_{\mathcal{B}}\left(x_{i}\right)\right]
\end{gathered}
$$

Step 4:

Correlation coefficient of the, $\mathcal{A}, \mathcal{B} \in$ IFSs are specified in the rule

$$
\mathcal{P}_{z l}(\mathcal{A}, \mathcal{B})=\frac{C_{z l}(\mathcal{A}, \mathcal{B})}{\sqrt{C_{z l}(\mathcal{A}, \mathcal{A}) C_{z l}(\mathcal{B}, \mathcal{B})}}
$$

Step 5:

Order entire alternatives $\mathcal{A}_{i}(i=1,2, \ldots m)$ to choose most excellent obtained on step 4

\section{Problem projected by choice maker:}

Consider the following initial Value Problem using modified Euler-Cauchy method and Heun method $y^{\prime}=\mathrm{t}+$ $y, y(1)=0$ with $\mathrm{h}=0.2$ on the interval [1,2] and the exact solution is given by $(t)=\frac{1}{1+t^{2}}$.

The modified Euler-Cauchy method are specified with $y_{j+1}=y_{j}+k_{2}$

In which $\mathrm{k}_{1}=\mathrm{hf}\left(\mathrm{t}_{\mathrm{j}}, \mathrm{y}_{\mathrm{j}}\right) \mathrm{k}_{2}=\mathrm{hf}\left(\mathrm{t}+\frac{h}{2^{3}}, \mathrm{y}_{\mathrm{j}}+\frac{k_{1}}{2}\right)$

For $\mathrm{j}=0, \mathrm{~h}=0.2, \mathrm{t}_{0}=1, \mathrm{y}_{0}=0$.

$$
\mathrm{K}_{1}=0.2(1+0)=0.2
$$$$
\mathrm{K}_{2}=0.2\left(1+0.1+0.1+\frac{0.2}{2}\right)=0.24
$$

$$
\mathrm{y}(1.2)=y_{1}=y_{0+k_{2}}=0+0.24=0.24
$$

Similarly for $\mathrm{j}=1,2,3$, 4 we have

$$
\begin{gathered}
y(1.4)=y_{2}=0.5768 \\
y(1.6)=y_{3}=1.031696 \\
y(1.8)=y_{4}=1.63066912
\end{gathered}
$$

Modified Euler - Cauchy method

pproximate solution with $h=$ 0.2

\begin{tabular}{|l|c|c|}
\hline $\mathrm{X}$ & $\begin{array}{c}\text { Approximation of } \\
\text { modified Euler Cauchy } \\
\text { method }\end{array}$ & $\mathrm{W}=\frac{y}{\Sigma y}$ \\
\hline 1.2 & 0.24 & 0.068982066594183 \\
\hline 1.4 & 0.5768 & 0.165786900048021 \\
\hline 1.6 & 1.031696 & 0.296535509070636 \\
\hline 1.8 & 1.63066912 & 0.468695524287160 \\
\hline
\end{tabular}

Exact Solution

Table-2: The values of Exact solution with $h=0.2$

\begin{tabular}{|l|c|c|}
\hline $\mathrm{X}$ & Exact solution & $\mathrm{W}=\frac{y}{\sum y}$ \\
\hline 1.2 & 0.409836066 & 0.324129220988464 \\
\hline 1.4 & 0.337837838 & 0.267187600935460 \\
\hline 1.6 & 0.280898876 & 0.222155982373731 \\
\hline 1.8 & 0.235849057 & 0.186527193329720 \\
\hline
\end{tabular}

Heun Method: 
Table-3: The values of approximate solution with $h=$ 0.2

\begin{tabular}{|l|c|c|}
\hline $\mathrm{X}$ & $\begin{array}{c}\text { Approximation of Heun } \\
\text { method }\end{array}$ & $\mathrm{W}=\frac{y}{\Sigma y}$ \\
\hline 1.2 & 0.24 & 0.084072748485556 \\
\hline 1.4 & 0.4084 & 0.143063793672920 \\
\hline 1.6 & 0.826248 & 0.289437251211222 \\
\hline 1.8 & 1.38002256 & 0.483426206630302 \\
\hline
\end{tabular}

Numerical Illustration

An HDFC Mutual Fund plans to invest money in a best company. Four factors are discussed to invest on one company

$\mathrm{M}_{1}$ : Hindustan Unilever Limited

$\mathrm{M}_{2}$ : WIPRO

$\mathrm{M}_{3}$ : Bharat Heavy Electricals Limited

$\mathrm{M}_{4}$ : SPICE JET

$\mathrm{M}_{5}$ : TATA Motors

Four factors are discussed to invest on one company
$\mathrm{F}_{1}$ : Opinion of Consumer

$\mathrm{F}_{2}$ : Growth feature

$\mathrm{F}_{3}$ : Profit and Loss analysis

$\mathrm{F}_{4}$ : Trust on the company

The alternates of five companies $M_{i}$ for $i=1,2,3,4,5$, are to be figured out by three decision makers, and the weighting vector are obtained by normalizing for solution of Euler - modified method by decision maker and the exact the solution $\mathrm{W}=(0.068982066594183,0.165786900048021,0.2965355090$ 70636 , $0.468695524287160)^{\mathrm{T}}$ $\omega=(0.324129220988464,0.267187600935460,0.222155982$ $373731,0.186527193329720)^{\mathrm{T}}$ under the above said four attributes weighting vector attained from normalizing in result of Heun method proposed by decision maker be $\mathrm{w}=(0.084072748485556,0.143063793672920,0.2894372512$ $11222,0.483426206630302)^{\mathrm{T}}$. Vague values in decision matrices $\mathcal{R}_{\mathrm{k}}=\left({\overline{r_{l \jmath}}}^{(k)}\right)_{5 \times 4}$ for $\mathrm{k}=1,2,3,4 \mathrm{j}=1,2,3,4$.

$$
\begin{aligned}
\mathcal{R}^{(1)}= & {\left[\begin{array}{llll}
{[0.5140,0.3645]} & {[0.5463,0.3420]} & {[0.3025,0.4563]} & {[0.6045,0.2734]} \\
{[0.4916,0.3701]} & {[0.7515,0.1430]} & {[0.6173,0.2615]} & {[0.1742,0.6217]} \\
{[0.3869,0.4042]} & {[0.8126,0.1340]} & {[0.5436,0.2342]} & {[0.3916,0.3245]} \\
{[0.2678,0.6140]} & {[0.5236,0.3213]} & {[0.6454,0.1326]} & {[0.4135,0.4158]} \\
{[0.6471,0.2305]} & {[0.4322,0.3341]} & {[0.5543,0.1426]} & {[0.6520,0.2513]}
\end{array}\right] } \\
\mathcal{R}^{(2)}= & {\left[\begin{array}{lllll}
{[0.2435,0.4326]} & {[0.4301,0.1653]} & {[0.5932,0.3014]} & {[0.3851,0.2942]} \\
{[0.5437,0.3017]} & {[0.7160,0.1543]} & {[0.3562,0.4125]} & {[0.1906,0.6143]} \\
{[0.7510,0.1231]} & {[0.2345,0.4314]} & {[0.4265,0.2241]} & {[0.6247,0.1732]} \\
{[0.2663,0.6024]} & {[0.1563,0.5422]} & {[0.7051,0.1872]} & {[0.5945,0.3132]} \\
{[0.4532,0.2541]} & {[0.4301,0.3362]} & {[0.5012,0.2537]} & {[0.1268,0.4534]}
\end{array}\right] } \\
\mathcal{R}^{(3)}= & {\left[\begin{array}{lllll}
{[0.3451,0.2543]} & {[0.4173,0.4650]} & {[0.4263,0.1753]} & {[0.2947,0.5436]} \\
{[0.5237,0.2343]} & {[0.7162,0.2145]} & {[0.5532,0.1523]} & {[0.7562,0.2317]} \\
{[0.4263,0.3827]} & {[0.2315,0.5634]} & {[0.2645,0.4332]} & {[0.3456,0.3544]} \\
{[0.2517,0.6432]} & {[0.3941,0.3137]} & {[0.5417,0.3462]} & {[0.5723,0.2462]} \\
{[0.3362,0.2537]} & {[0.2245,0.7130]} & {[0.3627,0.4352]} & {[0.1762,0.5234]}
\end{array}\right] } \\
\mathcal{R}^{(4)}= & {\left[\begin{array}{lllll}
{[0.8123,0.1045]} & {[0.1344,0.6542]} & {[0.6543,0.2217]} & {[0.2457,0.4322]} \\
{[0.4627,0.3354]} & {[0.3849,0.4021]} & {[0.3718,0.4321]} & {[0.4316,0.3253]} \\
{[0.5612,0.2316]} & {[0.5647,0.2334]} & {[0.5725,0.1244]} & {[0.1467,0.5372]} \\
{[0.2516,0.6243]} & {[0.7163,0.1436]} & {[0.2247,0.5332]} & {[0.5238,0.1276]} \\
{[0.3612,0.4217]} & {[0.2537,0.3442]} & {[0.7106,0.1623]} & {[0.6232,0.2314]}
\end{array}\right] }
\end{aligned}
$$

$r_{5}^{(1)}=(0.623415315262261,0.272249703400927)$

$r_{1}^{(2)}=(0.497460337283030,0.341165900827861)$

$r_{2}^{(2)}=(0.597573342596660,0.441069115786626)$

$r_{1}^{(1)}=(0.556738642625661,0.392840996908038)$

$r_{2}^{(1)}=(0.654495670880774,0.41725478776274)$

$r_{3}^{(1)}=(0.678116204829784,0.320584782458808)$

$r_{4}^{(1)}=(0.557701466085131,0.442002450458036)$ 
$r_{3}^{(2)}=(0.651059184531906,0.280064612166415)$

$r_{4}^{(2)}=(0.594671314367032,0.483301260770892)$

$r_{5}^{(2)}=(0.455335789839936,0.362143085318801)$

$r_{1}^{(3)}=(0.402404213243860,0.423217272954025)$

$r_{2}^{(3)}=(0.704690734659643,0.223395008380610)$

$r_{3}^{(3)}=(0.365721049072416,0.471164932863658)$

$r_{4}^{(3)}=(0.519300211482871,0.444739041227247)$

$r_{5}^{(3)}=(0.321715461752578,0.558182323397629)$

$r_{1}^{(4)}=(0.685182856907631,0.426054432025601)$

$r_{2}^{(4)}=(0.435215974367214,0.397714931881212)$

$r_{3}^{(4)}=(0.547259681436743,0.329895377926928)$

$r_{4}^{(4)}=(0.583591909218945,0.418506280435369)$

$r_{5}^{(4)}=(0.619044756325626,0.336538497201658)$

Step 2:

By Numerical methods using Euler - Cauchy method the weights are defined .

$$
\begin{gathered}
\mathrm{W}=(0.084072748485556,0.143063793672920,0.2894372 \\
51211222,0.483426206630302)^{\mathrm{T}}
\end{gathered}
$$

Utilizing IFHA operator for the matrix obtained in step 1

$$
\begin{aligned}
& r_{1}=(0.500133011521247,0.267724298507896) \\
& r_{2}=(0.600936103061978,0.350140093239254) \\
& r_{3}=(0.592745029237981,0.280282629895632) \\
& r_{4}=(0.533671417515458,0.408896992402703) \\
& r_{5}=(0.506764312312543,0.280246279414072)
\end{aligned}
$$

Step 3 \& step 4:

Correlation coefficient of IFS $\mathcal{A}, \mathcal{B}$ is specified as

$$
\begin{gathered}
\mathcal{P}_{z l}(\mathcal{A}, \mathcal{B})=\frac{C_{z l}(\mathcal{A}, \mathcal{B})}{\sqrt{C_{z l}(\mathcal{A}, \mathcal{A}) C_{z l}(\mathcal{B}, \mathcal{B})}} \\
\mathcal{P}_{z l}\left(r_{1}, \widetilde{r_{1}}\right)=0.436784730372289 \\
\mathcal{P}_{z l}\left(r_{2}, \widetilde{r_{2}}\right)=0.502194513950827 \\
\mathcal{P}_{z l}\left(r_{3}, \widetilde{r_{3}}\right)=0.419677426702777 \\
\mathcal{P}_{z l}\left(r_{4}, \widetilde{r_{4}}\right)=0.605989275744390 \\
\mathcal{P}_{z l}\left(r_{5}, \widetilde{r_{5}}\right)=0.454193869849264
\end{gathered}
$$

Step 5:

Rank alternatives $\mathrm{M}_{\mathrm{i}}(\mathrm{i}=1,2,3,4,5)$ in accordance with the correlation coefficient $\mathcal{P}_{z l}(\mathcal{A}, \mathcal{B})$ is given by $M_{4}>M_{2}>M_{5}>M_{1}>M_{3}$

Thus the optimum choice is $\mathrm{M}_{4}$.

\section{CONCLUSION:}

Here Euler, modified Euler and Heun method are used to calculate the solutions of decision maker weights. The weights of attribute are in the type of actual numbers.
Values of attribute being the formation of Intuitionistic Fuzzy Values. Combined decision matrix is obtained by the operators IFOWA and IFHA which aggregates all Individual Intuitionistic Fuzzy Decision Matrices provided by the decision makers. Correlation coefficient is used for ranking the alternatives. Finally the most desirable alternative is choosen.

\section{REFERENCES:}

1. Atanassov,K.(1986). Intuitionistic Fuzzy sets, Fuzzy sets and Systems, 20,87-96.

2. Atanassov.K.(1994) operators over interval valued intuitionistic fuzzy ses,Fuzzy Sets and Systems, 64,159174.

3. Atanassov, K., (1999). Intuitionistic Fuzzy Sets: Theory and Applications. Physica-Verlag, Heidelberg, New York.

4. Gerstenkorn , T., \& Manko. J. (1991), Correlation of Intuitionistic Fuzzy sets, Fuzzy Sets and Systems, 44,39-43.

5. Mathews, J.H, \& Fink, K.D. (2012). Numerical Methods using MATLAB PHI Learning Private Limited New Delhi.

6. Jeeva, S., Robinson, J.P.: Application of Sumudu Transform in Intuitionistic Fuzzy MAGDM Problems, International Journal of Pure and Applied Mathematics, 119(11), 109-117 (2017)

7. M.K.Jain S.R.K.Iyengar \& R.K.Jain Numerical Methods for Scientific and Engineering Computation New age International (P) LTD, Publishers New Delhi(1999)

8. Robinson, J.P, \& Jeeva, S. Application of Double Sumudu Transform in MAGDM problems with Intuitionistic Triangular Fuzzy Sets, International Journal of Research in Advent Technology, 6(7), 1620-1628 (2018)

9. Robinson, J.P, \& Indhumathi, M, Application of Singularly perturbed Delay Differential Equations in Intuitionistic Fuzzy MAGDM Problems, International Journal of Research in Advent Technology, 6(7), 1384-1394 (2018)

10. Robinson .J.P. \& Amirtharaj, E.C.H.,(2012). Vague Correlation coefficient of Interval Vague sets, International Journal of Fuzzy System Applications, 2(1),18 -34h

11. Robinson .J.P. \& Amirtharaj, E.C.H.,(2014). Efficient Multiple Attribute Group Decision Making models with Correlation coefficient of Vague sets, International Journal of Operations Research and Information Systems, 5(3), 2749.

12. Kingston .J , Robinson. J.P \& Dhayabaran, D.P., (2016) MAGDM Problems using Runge-Kutta Method of Harmonic Mean, Aryabhatta Journal of Mathematics and Informatics Vol.8,Issue 2, July - Dec, 20160975 - 7135.

13. Xu Z.S. (2005). An Overview of Methods for Determining OWA Weights, International Journal of Intelligent Systems, 20, 843-865.

14. Xu, Z.S., \& Yager, R.R. Some geometric aggregation operators based on Intuitionistic Fuzzy sets. International Journal of General Systems, 35, 417-433 (2006)

15. Yager ,R.R.(1988), On ordered weighted aggregation operators in multi-criteria decision making, IEEE Transcations on Systems, Man, and Cybernetics, 18, 183 190

16. Yager ,R.R (2004). Generalized OWA aggregation operators, Fuzzy Optimization and Decision Making 3,93107.

17. Yager, R.R., \& Filev, D.P. Induced ordered weighted averaging operators, IEEE Transactions on Systems, Man, and Cybernetics Part B, 29, 141-150 (1999) 